\title{
m - projective curvature tensor on a Lorentzian para - Sasakian manifolds
}

\author{
Amit Prakash ${ }^{1}$, Mobin Ahmad ${ }^{2}$ and Archana Srivastava ${ }^{3}$ \\ ${ }^{1}$ Department of Mathematics, National Institute of Technology, Kurukshetra, \\ Haryana - 136119, India. \\ ${ }^{2}$ Department of Mathematics, Integral University, Kursi Road Lucknow - 226 026, India, \\ ${ }^{3}$ Department of Mathematics, S. R. Institute of Management and Technology, BKT, Lucknow - 227 \\ 202, India,
}

Abstract: In this paper we studied m-projectively flat, $m$-projectively conservative, $\varphi$-m-projectively flat $L P$ Sasakian manifold. It has also been proved that quasi $m$ - projectively flat LP-Sasakian manifold is locally isometric to the unit sphere $S^{n}(1)$ if and only if $M^{n}$ is m-projectively flat.

Keywords - Einstein manifold, m-projectively flat, m-projectively conservative, quasi m-projectively flat, $\varphi$-m-projectively flat.

\section{Introduction}

The notion of Lorentzian para contact manifold was introduced by K. Matsumoto. The properties of Lorentzian para contact manifolds and their different classes, viz LP-Sasakian and LSP-Sasakian manifolds have been studied by several authors. In [13], M.Tarafdar and A. Bhattacharya proved that a LP-Sasakian manifold with conformally flat and quasi - conformally flat curvature tensor is locally isometric with a unit sphere $S^{n}(1)$. Further, they obtained that an LP-Sasakian manifold with $R(X, Y) \cdot \tilde{C}=0$ is locally isometric with a unit sphere $S^{n}(1)$, where $\widetilde{C}$ is the conformal curvature tensor of type $(1,3)$ and $R(X, Y)$ denotes the derivation of tensor of tensor algebra at each point of the tangent space. J.P. Singh [10] proved that an $\mathrm{m}$ projectively flat para-Sasakian manifold is an Einstein manifold. He has also shown that if in an Einstein PSasakian manifold $R(\xi, X) \cdot W=0$ holds, then it is locally isometric with a unit sphere $H^{n}(1)$. Also an $\mathrm{n}$ dimensional $\eta$-Einstien P-Sasakian manifold satisfying $W(\xi, X) \cdot R=0$ if and only if either manifold is locally isometric to the hyperbolic space $H^{n}(-1)$ or the scalar curvature tensor $r$ of the manifold is $-n(n-1)$. S.K. Chaubey [18], studied the properties of m-projective curvature tensor in LP-Sasakian, Einstein LP-Sasakian and $\eta$-Einstien LP-Sasakian manifold. LP-Sasakian manifolds have also studied by Matsumoto and Mihai [4], Takahashi [11], De, Matsumoto and Shaikh [2], Prasad \& De [9], Venkatesha and Bagewadi[14].

In this paper, we studied the properties of LP-Sasakian manifolds equipped with m-projective curvature tensor. Section 1 is introductory. Section 2 deals with brief account of Lorentzian para-Sasakian manifolds. In section 3, we proved that an m-projectively flat LP-Sasakian manifold is an Einstein manifold and an LP-Sasakian manifold satisfying $\left(C_{1}^{1} W\right)(Y, Z)=0$ is of constant curvature is m-projectively flat. In section 4, we proved that an Einstein LP-Sasakian manifold is m-projectively conservative if and only if the scalar curvature is constant. In section 5 , we proved that an $\mathrm{n}$-dimensional $\varphi$-m-projectively flat LP-Sasakian manifold is an $\eta$-Einstein manifold. In last, we proved that an $\mathrm{n}$-dimensional quasi $\mathrm{m}$ - projectively flat LP-Sasakian manifold $M^{n}$ is locally isometric to the unit sphere $S^{n}(1)$ if and only if $M^{n}$ is m-projectively flat.

\section{Preliminaries}

An n- dimensional differentiable manifold $M^{n}$ is a Lorentzian para-Sasakian (LP-Sasakian) manifold, if it admits a $(1,1)$ - tensor field $\phi$, a vector field $\xi$, a 1-form $\eta$ and a Lorentzian metric $g$ which satisfy

$$
\begin{gathered}
\phi^{2} X=X+\eta(X) \xi \\
\eta(\xi)=-1 \\
g(\phi X, \phi Y)=g(X, Y)+\eta(X) \eta(Y) \\
g(X, \xi)=\eta(X) \\
\left(D_{X} \phi\right)(Y)=g(X, Y) \xi+\eta(Y) X+2 \eta(X) \eta(Y) \xi \\
\text { and } D_{X} \xi=\phi X
\end{gathered}
$$

for arbitrary vector fields $\mathrm{X}$ and $\mathrm{Y}$, where $\mathrm{D}$ denote the operator of covariant differentiation with respect to Lorentzian metric g, (Matsumoto, (1989) and Matsumoto and Mihai, (1988)). In an LP-Sasakian manifold $M^{n}$ with structure $(\phi, \xi, \eta, g)$, it is easily seen that 
$\begin{array}{lll}\text { (a) } \phi \xi=0 & \text { (b) } \eta(\phi X)=0 & \text { (c) rank } \phi=(n-1)\end{array}$

Let us put $F(X, Y)=g(\phi X, Y)$,

then the tensor field $F$ is symmetric $(0,2)$ tensor field

$$
F(X, Y)=F(Y, X) \text {, }
$$

$F(X, Y)=\left(D_{X} \eta\right)(Y)$

and $\left(D_{X} \eta\right)(Y)-\left(D_{Y} \eta\right)(X)=0$.

An LP- Sasakian manifold $M^{n}$ is said to be Einstein manifold if its Ricci tensor $\mathrm{S}$ is of the form $S(X, Y)=k g(X, Y)$.

An LP- Sasakian manifold $M^{n}$ is said to be an $\eta$-Einstein manifold if its Ricci tensor $\mathrm{S}$ is of the form $S(X, Y)=\alpha g(X, Y)+\beta \eta(X) \eta(Y)$,

for any vector fields $\mathrm{X}$ and $\mathrm{Y}$, where $\alpha, \beta$ are the functions on $M^{n}$.

Let $M^{n}$ be an $n$-dimensional LP-Sasakian manifold with structure $(\varphi, \xi, \eta, g)$. Then we have

(Matsumoto and Mihai, (1998) and Mihai, Shaikh and De,(1999)).

$$
\begin{gathered}
g(R(X, Y) Z, \xi)=\eta(R(X, Y) Z)=g(Y, Z) \eta(X)-g(X, Z) \eta(Y) \\
R(\xi, X) Y=g(X, Y) \xi-\eta(Y) X, \\
R(\xi, X) \xi=X+\eta(X) \xi, \\
R(X, Y) \xi=\eta(Y) X-\eta(X) Y, \\
S(X, \xi)=(n-1) \eta(X), \\
S(\varphi X, \varphi Y)=S(X, Y)+(n-1) \eta(X) \eta(Y),
\end{gathered}
$$

for any vector fields $X, Y, Z$; where $R(X, Y) Z$ is the Riemannian curvature tensor of type $(1,3) . S$ is a Ricci tensor of type $(0,2), Q$ is Ricci tensor of type $(1,1)$ and $r$ is the scalar curvature.

$g(Q X, Y)=S(X, Y)$ for all $X, Y$.

m-projective curvature tensor $W$ on an Riemannian manifold $\left(M^{n}, g\right)(n>3)$ of type $(1,3)$ is defined as follows (G.P.Pokhariyal and R.S. Mishra (1971)).

$$
W(X, Y) Z=R(X, Y) Z-\frac{1}{2(n-1)}[S(Y, Z) X-S(X, Z) Y+g(Y, Z) Q X-g(X, Z) Q Y],
$$

so that $W(X, Y, Z, U) \stackrel{\text { def }}{=} g(W(X, Y) Z, U)=' W(Z, U, X, Y)$.

On an $n$ - dimensional LP-Sasakian manifold, the Concircular curvature tensor $C$ is defined as

$$
C(X, Y) Z=R(X, Y) Z-\frac{r}{n(n-1)}[g(Y, Z) X-g(X, Z) Y] .
$$

Now, in view of $S(X, Y)=\frac{r}{n} g(X, Y),(2.18)$ becomes

$$
W(X, Y) Z=C(X, Y) Z \text {. }
$$

Thus, in an Einstein LP-Sasakian manifold, m-projective curvature tensor $W$ and the concircular curvature tensor $C$ concide.

\section{III. m-projectively flat LP-Sasakian manifold}

In this section we assume that $W(X, Y) Z=0$.

Then from (2.18), we get

$$
R(X, Y) Z=\frac{1}{2(n-1)}[S(Y, Z) X-S(X, Z) Y+g(Y, Z) Q X-g(X, Z) Q Y] .
$$

Contracting (3.1) with respect to $X$, we get

$$
S(Y, Z)=\frac{r}{n} g(Y, Z) \text {. }
$$

Hence we can state the following theorem.

Theorem 3.1: Let $M^{n}$ be an $n$-dimensional m-projectively flat LP-Sasakian manifold, then $M^{n}$ be an Einstien manifold.

Contracting (2.18) with respect to $X$, we get

$$
\begin{aligned}
& \quad\left(C_{1}^{1} W\right)(Y, Z)=S(Y, Z)-\frac{1}{2(n-1)}[n S(Y, Z)-S(Y, Z)+\operatorname{rg}(Y, Z)-g(Y, Z)], \\
& \text { Or, }\left(C_{1}^{1} W\right)(Y, Z)=\frac{n}{2(n-1)}\left[S(Y, Z)-\frac{r}{n} g(Y, Z)\right],
\end{aligned}
$$

where $\left(C_{1}^{1} W\right)(Y, Z)$ is the contraction of $W(X, Y) Z$ with respect to $X$.

If $\left(C_{1}^{1} W\right)(Y, Z)=0$, then from (3.4), we get

$$
S(Y, Z)=\frac{r}{n} g(Y, Z) \text {. }
$$

Using (3.5) in (3.1), we get

$$
' R(X, Y, Z, W)=\frac{r}{n(n-1)}[g(Y, Z) g(X, W)-g(X, Z) g(Y, W)] .
$$

Hence we can state the following theorem.

Theorem 3.2. An m-projectively flat LP-Sasakian manifold satisfying $\left(C_{1}^{1} W\right)(Y, Z)=0$ is a manifold of constant curvature. 
Using (3.5) and (3.6) in (2.18), we get

$$
W(X, Y) Z=0 \text {, }
$$

i.e. the manifold $M^{n}$ is m-projectively flat.

Hence we can state the following theorem.

Theorem 3.3. An LP-Sasakian manifold $\left(M^{n}, g\right)(n>3)$ satisfying $\left(C_{1}^{1} W\right)(Y, Z)=0$, is of constant curvature is $\mathrm{m}$-projectively flat.

\section{Einstein LP-Sasakian manifold satisfying $(\operatorname{div} W)(X, Y) Z=0$}

Definition 4.1. A manifold $\left(M^{n}, g\right)(n>3)$ is called m-projectively conservative if (Hicks N.J.(1969)),

$$
\operatorname{div}(W)=0 \text {, }
$$

where div denotes divergence.

Now differentiating (2.18) covariently, we get

$$
\begin{aligned}
\left(D_{U} W\right)(X, Y) Z= & \left(D_{U} R\right)(X, Y) Z-\frac{1}{2(n-1)}\left[\left(D_{U} S\right)(Y, Z) X-\left(D_{U} S\right)(X, Z) Y+g(Y, Z)\left(D_{W} Q\right) X\right. \\
& \left.-g(X, Z)\left(D_{W} Q\right) Y\right] .
\end{aligned}
$$

Which gives on contraction

$$
\begin{aligned}
\operatorname{div}(W)(X, Y) Z= & \operatorname{div}(R)(X, Y) Z-\frac{1}{2(n-1)}\left[\left(D_{X} S\right)(Y, Z)-\left(D_{Y} S\right)(X, Z)+g(Y, Z) \operatorname{div}(Q) X-\right. \\
& g X, Z \operatorname{div} Q Y .
\end{aligned}
$$

But $\operatorname{div}(Q)=\frac{1}{2} d r$, using in (4.3), we get

$$
\begin{aligned}
\operatorname{div}(W)(X, Y) Z= & \operatorname{div}(R)(X, Y) Z-\frac{1}{2(n-1)}\left[\left(D_{X} S\right)(Y, Z)-\left(D_{Y} S\right)(X, Z)+\frac{1}{2} g(Y, Z) d r(X)-\right. \\
& 12 g X, Z d r Y .
\end{aligned}
$$

But from (Eisenhart L.P.(1926)), we have

Using (4.5) in (4.4), we get

$$
\operatorname{div}(R)(X, Y) Z=\left(D_{X} S\right)(Y, Z)-\left(D_{Y} S\right)(X, Z) .
$$

From (4.6) and (4.7), we get

From (4.1) and (4.8), we get

$$
\operatorname{div}(W)(X, Y) Z=-\frac{1}{4(n-1)}[g(Y, Z) d r(X)-g(X, Z) d r(Y)] .
$$

$$
[g(Y, Z) d r(X)-g(X, Z) d r(Y)]=0,
$$

which shows that $r$ is constant. Again if $r$ is constant then from (4.8), we get

$$
\operatorname{div}(W)(X, Y) Z=0 .
$$

Hence we can state the following theorem.

Theorem 4.1. An Einstein LP-Sasakian manifold $\left(M^{n}, g\right)(n>3)$ is m-projectively conservative if and only if the scalar curvature is constant.

\section{V. $\varphi$ - m-projectively flat LP-Sasakian manifold}

Definition 5.1. A differentiable manifold $\left(M^{n}, g\right), n>3$, satisfying the condition

$$
\varphi^{2} W(\varphi X, \varphi Y) \varphi Z=0,
$$

is called $\varphi$-m - projectively flat LP-Sasakian manifold.(Cabrerizo, Fernandez, Fernandez and Zhen (1999)).

Suppose that $\left(M^{n}, g\right), n>3$ is a $\varphi-\mathrm{m}$ - projectively flat LP-Sasakian manifold. It is easy to see that $\varphi^{2} W(\varphi X, \varphi Y) \varphi Z=0$, holds if and only if

for any vector fields $X, Y, Z, W$.

$$
g(W(\varphi X, \varphi Y) \varphi Z, \varphi W)=0,
$$

By the use of (2.18), $\varphi-\mathrm{m}$ - projectively flat means

$$
\begin{aligned}
& \text { 'R( }(\varphi X, \varphi Y, \varphi Z, \varphi W)=\frac{1}{2(n-1)}\left[\begin{array}{c}
S(\varphi Y, \varphi Z) g(\varphi X, \varphi W)-S(\varphi X, \varphi Z) g(\varphi Y, \varphi W)+ \\
g(\varphi Y, \varphi Z) S(\varphi X, \varphi W)-g(\varphi X, \varphi Z) S(\varphi Y, \varphi W)
\end{array}\right], \\
& \text { where ' } R(X, Y, Z, W)=g(R(X, Y) Z, W) .
\end{aligned}
$$


Let $\left\{e_{1}, e_{2}, \ldots, e_{n-1}, \xi\right\}$ be a local orthonormal basis of vector fields in $M^{n}$ by using the fact that $\left\{\varphi e_{1}, \varphi e_{2}, \ldots, \varphi e_{n-1}, \xi\right\}$ is also a local orthonormal basis, if we put $X=W=e_{i}$ in (5.2) and sum up with respect to $i$, then we have

$$
\begin{aligned}
\sum_{i=1}^{n-1} R\left(\varphi e_{i}, \varphi Y, \varphi Z, \varphi e_{i}\right) & =\frac{1}{2(n-1)} \sum_{i=1}^{n-1}\left[S(\varphi Y, \varphi Z) g\left(\varphi e_{i}, \varphi e_{i}\right)-S\left(\varphi e_{i}, \varphi Z\right) g\left(\varphi Y, \varphi e_{i}\right)\right. \\
& \left.+g(\varphi Y, \varphi Z) S\left(\varphi e_{i}, \varphi e_{i}\right)-g\left(\varphi e_{i}, \varphi Z\right) S\left(\varphi Y, \varphi e_{i}\right)\right] .
\end{aligned}
$$

On an LP-Sasakian manifold, we have (Ö̈zgür (2003))

$$
\begin{aligned}
& \sum_{i=1}^{n-1} R\left(\varphi e_{i}, \varphi Y, \varphi Z, \varphi e_{i}\right)=S(\varphi Y, \varphi Z)+g(\varphi Y, \varphi Z), \\
& \sum_{i=1}^{n-1} S\left(\varphi e_{i}, \varphi e_{i}\right)=r+(n-1), \\
& \sum_{i=1}^{n-1} g\left(\varphi e_{i}, \varphi Z\right) S\left(\varphi Y, \varphi e_{i}\right)=S(\varphi Y, \varphi Z), \\
& \sum_{i=1}^{n-1} g\left(\varphi e_{i}, \varphi e_{i}\right)=(n+1), \\
& \sum_{i=1}^{n-1} g\left(\varphi e_{i}, \varphi Z\right) g\left(\varphi Y, \varphi e_{i}\right)=g(\varphi Y, \varphi Z),
\end{aligned}
$$

so, by the virtue of (5.4)-(5.8), the equation(5.3) takes the form

$$
S(\varphi Y, \varphi Z)=\left[\frac{r}{n-1}-1\right] g(\varphi Y, \varphi Z)
$$

By making the use of (2.3) and (2.17) in (5.9), we get

$$
S(Y, Z)=\left[\frac{r}{n-1}-1\right] g(Y, Z)+\left[\frac{r}{n-1}-n\right] \eta(Y) \eta(Z) .
$$

Hence we can state the following theorem.

Theorem 5.1. Let $M^{n}$ be an $n$-dimenstional $n>3, \varphi-\mathrm{m}$ - projectively flat LP-Sasakian manifold, then $M^{n}$ is an $\eta$-Einstein manifold with constants $\alpha=\left[\frac{r}{n-1}-1\right]$ and $\beta=\left[\frac{r}{n-1}-n\right]$.

\section{VI. quasi m-projectively flat LP-Sasakian manifold}

Definition 6.1. An LP-Sasakian manifold $M^{n}$ is said to be quasi m-projectively flat, if for any vector fields $X, Y, Z, U$.

$$
g(W(X, Y) Z, \varphi U)=0,
$$

From (2.18), we get

$$
\begin{aligned}
g(W(X, Y) Z, \varphi U) & =g(R(X, Y) Z, \varphi U)-\frac{1}{2(n-1)}[S(Y, Z) g(X, \varphi U)-S(X, Z) g(Y, \varphi U) \\
& +g(Y, Z) S(X, \varphi U)-g(X, Z) S(Y, \varphi U)] .
\end{aligned}
$$

Let $\left\{e_{1}, e_{2}, \ldots, e_{n-1}, \xi\right\}$ be a local orthonormal basis of vector fields in $M^{n}$ by using the fact that $\left\{\varphi e_{1}, \varphi e_{2}, \ldots, \varphi e_{n-1}, \xi\right\}$ is also a local orthonormal basis, if we put $X=\varphi e_{i}, U=e_{i}$ in (5.2) and sum up with respect to $i$, then we have

$$
\begin{aligned}
& \sum_{i=1}^{n-1} g\left(W\left(\varphi e_{i}, Y\right) Z, \varphi e_{i}\right)=\sum_{i=1}^{n-1} g\left(R\left(\varphi e_{i}, Y\right) Z, \varphi e_{i}\right)-\frac{1}{2(n-1)} \sum_{i=1}^{n-1}\left[S(Y, Z) g\left(\varphi e_{i}, \varphi e_{i}\right)-\right. \\
&\left.S\left(\varphi e_{i}, Z\right) g\left(Y, \varphi e_{i}\right)+g(Y, Z) S\left(\varphi e_{i}, \varphi e_{i}\right)-g\left(\varphi e_{i}, Z\right) S\left(Y, \varphi e_{i}\right)\right] .
\end{aligned}
$$

On an LP-Sasakian manifold by straight forward calculation, we get

$$
\begin{gathered}
\sum_{i=1}^{n-1} R\left(e_{i}, Y, Z, e_{i}\right)=\sum_{i=1}^{n-1} ' R\left(\varphi e_{i}, Y, Z, \varphi e_{i}\right)=S(Y, Z)+g(\varphi Y, \varphi Z), \\
\sum_{i=1}^{n-1} S\left(\varphi e_{i}, Z\right) g\left(Y, \varphi e_{i}\right)=S(Y, Z)-(n-1) \eta(Y) \eta(Z) \\
\text { Using (5.4), (5.7), (6.4), (6.5) in (6.3), we get } \\
\qquad \sum_{i=1}^{n-1} g\left(W\left(\varphi e_{i}, Y\right) Z, \varphi e_{i}\right)=S(Y, Z)+g(\varphi Y, \varphi Z)
\end{gathered}
$$




$$
-\frac{1}{2(n-1)}[(n-1) S(Y, Z)+(r+n-1) g(Y, Z)+2(n-1) \eta(Y) \eta(Z)]
$$

Using (2.3) in (6.6), we get

$$
\sum_{i=1}^{n-1} g\left(W\left(\varphi e_{i}, Y\right) Z, \varphi e_{i}\right)=\frac{1}{2}\left[S(Y, Z)-\left(\frac{r}{n}-1\right) g(Y, Z)\right] \text {. }
$$

If $M^{n}$ is quasi m-projectively flat, then (6.7) reduces to

$$
S(Y, Z)=\left(\frac{r}{n}-1\right) g(Y, Z) \text {. }
$$

Putting $Z=\xi$ in (6.8) and then using (2.6) and (2.16), we get

$$
r=n(n-1) \text {. }
$$

Using (6.9) in (6.8), we get

$$
S(Y, Z)=(n-1) g(Y, Z) \text {. }
$$

i.e. $M^{n}$ is an Einstein manifold.

Now using (6.10) in (2.18), we get

$$
W(X, Y) Z=R(X, Y) Z-[g(Y, Z) X-g(X, Z) Y] \text {. }
$$

If LP-Sasakian manifold is m-projectively flat, then from (6.11), we get $R(X, Y) Z=[g(Y, Z) X-g(X, Z) Y]$.

Hence we can state the following theorem.

Theorem 6.2. A quasi m-projectively flat LP-Sasakian manifold $M^{n}$ is locally isomeric to the unit sphere

$S^{n}(1)$ if and only if $M^{n}$ is m-projectively flat.

\section{References}

[1] D. E. Blair, Contact manifolds on Riemannian geometry, Lecture Notes in Mathematics, Vol.509, Spinger-Verlag, Berlin, 1976.

[2] U. C. De, K. Matsumoto and A. A. Shaikh, On Lorentzian para-Sasakian manifolds, Rendicontidel Seminario Mathematico di Messina, Series II, Supplemento al 3 (1999), 149-158.

[3] K. Matsumoto, On Lorentzian para-contact manifolds, Bull. Of Yamagata Univ. Nat. Sci., 12 (1989), 151-156.

[4] K. Matsumoto, I. Mihai, On certain transformation in a Lorentzian para-Sasakian manifold, Tensor N.S., 47 (1988), $189-197$.

[5] R. H. Ojha, A notes on the m-projective curvature tensor, Indian J. Pure Applied Math., 8 (1975), No. 12, 1531-1534.

[6] R. H. Ojha, On Sasakian manifold, Kyungpook Math. J., 13 (1973), 211-215.

[7] G. P. Pokhariyal and R. S. Mishra, Curvature tensor and their relativistic significance II, Yokohama Mathematical Journal, 19 (1971), 97-103

[8] S. Prasad and R. H. Ojha, Lorentzian para contact submanifolds, Publ. Math. Debrecen, 44/3-4 (1994), $215-223$.

[9] A. A. Shaikh and U. C. De, On 3-dimensional LP-Sasakian manifolds, Soochow J. of Math., 26 (4) (2000), $359-368$

[10] J. P. Singh, On an Einstein m-projective P-Sasakian manifolds, (2008) (to appear in Bull. Cal. Math. Soc.).

[11] T. Takahashi, Sasakian $\phi$-symmetric spaces, Tohoku Math. J., 29 (1977), 93-113.

[12] S. Tanno, Curvature tensors and non-existance of killing vectors, Tensor N. S., 2(1971), 387-394.

[13] M. Tarafdar and A. Bhattacharya, On Lorentzian para-Sasakian manifolds, Steps in Differential Geometry, Proceeding of the Colloquium of Differential Geometry, 25-30 july 2000, Debrecen, Hungry, 343-348

[14] Venkatesha and C. S. Begewadi, On concircular $\phi$-recurrent LP-Sasakian Manifolds, Differential Geometry-Dynamical Systems, 10 (2008), 312-319.

[15] K. Tano, and M. Kon, Structures on manifolds, Series in Pure Mathematics, Vol. 3, World Scientific, Singapore, 1984.

[16] A. Taleshian and N. Asghari, On LP-Sasakian manifolds satisfying certain conditions on the concircular curvature tensor, Differential Geometry- Dynamical Systems, 12,(2010), 228-232.

[17] S. K. Chaubey and R. H. Ojha, On the m-projective curvature tensor of a Kenmotsu manifold, Differential Geometry-Dynamical Systems, 12,(2010), 1-9.

[18] S. K. Chaubey, Some properties of LP-Sasakian manifolds equipped with m-projective curvature tensor, Bulletin of Mathematical Analysis and applications, 3 (4), (2011), 50-58. 